\title{
Analyse zootechnique et économique des systèmes d'élevage de porcs en Casamance (Sénégal)
}

\author{
Walter Ossebi ${ }^{1 *}$ Simplice Bosco Ayssiwede ${ }^{2}$ \\ Félix Nimbona ${ }^{3}$ Richard Malou ${ }^{4}$ Augustin Eric Djettin ${ }^{2}$ \\ Mariame Diop ${ }^{4}$ Ayao Missohou ${ }^{2}$ \\ Cet article est publié dans le prolongement du numéro thématique \\ «L'élevage porcin dans les pays tropicaux » (1-2) 2018.
}

\begin{abstract}
Mots-clés
Porcin, système d'exploitation agricole, commercialisation, zootechnie, Sénégal
\end{abstract}

Submitted: 14 July 2017

Accepted: 21 March 2019

Published: 20 May 2019

DOI: $10.19182 /$ remvt.31258.

\section{Résumé}

Afin de mieux cerner les caractéristiques zootechniques et économiques de l'élevage de porc, une étude diagnostique a été conduite de mars à juin 2016 auprès de 324 élevages porcins, sous forme d'enquêtes transversales et rétrospectives dans les trois régions de la Casamance au Sud Sénégal. Les résultats ont montré que l'élevage porcin était pratiqué à la fois par des hommes et des femmes mariés et instruits. Ils étaient majoritairement des ethnies Diola, Mancagne et Balante et associaient l'élevage à l'agriculture, à l'activité privée ou au commerce. Ils évoluaient dans trois systèmes d'élevage différents (traditionnel, semi-intensif ou intensif), avec une nette prédominance du système traditionnel $(85,5 \%$ des élevages). Ce dernier était caractérisé par des porcheries traditionnelles améliorées $(51,1 \%)$ ou des abris de fortune $(28,4 \%)$, où étaient surtout élevés les porcs de race locale $(90,4 \%$ des exploitations). La taille moyenne du cheptel était de $19,2 \pm 20,1$ porcs. Dans $97 \%$ des cas les éleveurs nourrissaient les animaux à partir de déchets de cuisine et de sous-produits agricoles et agro-industriels. L'âge moyen de mise à la reproduction était de 7,6 $\pm 1,8$ mois et la taille moyenne de la portée était de 7,4 $\pm 2,2$ porcelets. Ce sous-secteur d'élevage a un réel potentiel de réduction de la pauvreté car il génère selon les systèmes des revenus nets de 5435 à 7566 FCFA par porc engraissé par an. Néanmoins, le manque de formation des éleveurs, les habitats porcins inadaptés, les contraintes alimentaires et sanitaires restent les principaux facteurs handicapant l'essor de l'élevage porcin dans cette région. Il serait utile de trouver des voies alternatives d'amélioration des conditions d'habitat, d'alimentation et de suivi sanitaire des porcs, accompagnées d'une meilleure organisation et d'un renforcement de capacité des producteurs et des autres acteurs de la filière porcine.

- Comment citer cet article : Ossebi W., Ayssiwede S.B., Nimbona F., Malou R., Djettin A.E., Diop M., Missohou A., 2019. Zootechnical and economic analysis of pig farming systems in Casamance (Senegal). Rev. Elev. Med. Vet. Pays Trop., 72 (1): 13-22, doi: 10.19182/remvt.31258

\section{INTRODUCTION}

Dans les pays en développement, l'occupation des terres au profit de l'agriculture ou de l'urbanisation réduit considérablement les aires de pâturages des ruminants. Cet amenuisement de l'espace se fait dans un contexte d'accroissement numérique de ces animaux. Ces limites

1. Service d'économie rurale et gestion, Ecole inter-Etats des sciences

et médecine vétérinaires (EISMV), BP 5077, Dakar-Fann, Sénégal.

2. Service de zootechnie-alimentation, EISMV, Dakar-Fann, Sénégal.

3. Ministère de l'Environnement, de l'Agriculture et de l'Elevage (Mineagrie),

Centre national d'insémination artificielle et d'amélioration génétique (CNIAAG),

Gitega, Burundi.

4. Institut sénégalais de recherches agricoles (ISRA), Dakar, Sénégal.

* Auteur pour la correspondance

Tél. : +221338651008

Email : ossebi_3@yahoo.fr;w.ossebi@eismv.org

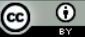

https://creativecommons.org/licenses/by/4.0/ font ressortir la place du porc, souvent délaissé alors qu'il est l'animal le plus consommé dans le monde (Cirad, 2007). L'élevage de porc produit sept à huit fois plus de viande que le bœuf par an et par tonne d'animaux sur pied en Afrique (Bathily, 1975). Il constitue une source importante de protéines et une activité secondaire pour plusieurs catégories socioprofessionnelles à qui il procure un revenu supplémentaire (Missohou et al., 2001 ; Mopaté Logténé et al., 2010 ; Ndeby et al., 2009 ; FAO, 2012a ; FAO, 2012b). Il représente ainsi un atout majeur pour la lutte contre l'insécurité alimentaire et l'amélioration des revenus des populations, particulièrement en Afrique où la pression démographique est de plus en plus forte. Comme le rapportent Mopaté Logténé et Koussou (2003), la mise en place d'un élevage de porc est le plus souvent motivée par le souhait du producteur de réaliser un projet et d'adopter une stratégie de diversification de la production pour lutter contre l'insécurité financière et alimentaire.

Le système d'élevage traditionnel est privilégié par les éleveurs pour la simplicité des techniques mises en œuvre et surtout pour la faiblesse des investissements consentis (Buldgen et al., 1994 ; Porphyre, 
2009). Les races indigènes, malgré leurs faibles performances, ont la faveur de ces exploitants en raison de leur faible prix d'achat, de leur rusticité, ainsi que de leur grande capacité d'adaptation et de valorisation des déchets et même de certaines plantes (FAO, 2012a).

Le Sénégal est un pays à forte majorité musulmane. Dans ce contexte, l'élevage porcin est mal considéré, même en Casamance où les chrétiens sont nombreux (Lalèyê, 2007). Avec une croissance démographique annuelle estimée à $3,2 \%$ à l'échelle du Sénégal, la production de viande de porc n'est pas suffisante pour couvrir les besoins des populations consommatrices. Le Sénégal est un pays où l'on consomme peu de viande : 13 kilogrammes par habitant par an contre 41,2 dans le monde toutes espèces confondues (Mankor, 2009 ; FAO, 2009). La production et la consommation de viande sont dominées par la viande rouge, $54 \%$ des viandes totales, dont $32 \%$ de bovins, $14 \%$ d'ovins et $8 \%$ de caprins (MEPA, 2015). La viande blanche représente $46 \%$ des viandes totales (dont $39 \%$ de volaille et $7 \%$ de porc). Cette consommation de porc (qui représente $15 \%$ de la viande blanche) au Sénégal est faite surtout par les chrétiens et expatriés. Les importations de viande de porc ne concernent que des marchés très spécifiques, notamment les charcuteries, avec des quantités très faibles, $1 \%$ de la production nationale (Niang et Mbaye, 2013 ; FAOSTAT, 2015 ; MEPA, 2015). De nombreuses études sur la filière porcine font ressortir qu'elle a été négligée dans les programmes nationaux d'appui à l'élevage et de développement rural (Le Glaumec, 2006 ; Seck, 2007). De plus, la plupart des pratiques rudimentaires décrites il y a plus de 15 ans restent en proportions très importantes (Buldgen et al., 1994 ; Missohou et al., 2001). L'objectif de cette étude était de mieux cerner, à travers une analyse diagnostique des systèmes de production porcine, les caractéristiques des systèmes d'élevage, les performances zootechniques et économiques ainsi que les contraintes liées au développement de l'élevage porcin dans la Casamance naturelle.

\section{MATERIEL ET METHODES}

\section{Zone d'étude}

L'étude a été réalisée en milieux urbain et rural des trois régions de la Casamance naturelle au sud du Sénégal, qui s'étend sur 28350 kilomètres carrés, soit un septième de la superficie du Sénégal (figure 1). Elle est limitée à l'ouest par l'océan Atlantique, à l'est par un affluent du fleuve Gambie, au nord par la Gambie et au sud par la Guinée Bissau. Son climat est de type soudanien avec une saison de pluie allant de juin à octobre et une saison sèche qui couvre le reste de l'année. Elle est la partie la plus arrosée du Sénégal avec une pluviométrie supérieure à $800 \mathrm{~mm}$ et on y retrouve $20 \%$ des terres arables du pays. Aussi, la densité du réseau hydrographique rend cette zone très propice à la riziculture (CSE, 2007). Ces conditions sont favorables au développement des activités d'élevage, notamment celui des porcs.

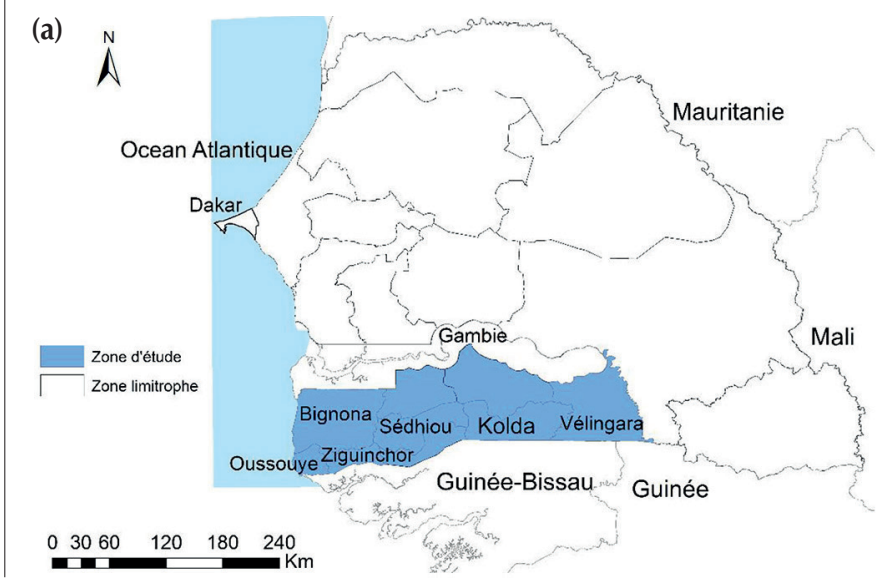


- le poids vif moyen à la vente était de $90 \mathrm{~kg}$ dans le système intensif, $80 \mathrm{~kg}$ dans le système semi-intensif et $40 \mathrm{~kg}$ dans le système traditionnel ;

- tous les éleveurs du système intensif payaient 70000 FCFA pour acheminer une tonne d'aliments industriels depuis Dakar une fois par an ;

- le rendement carcasse a été estimé à $75 \%$ pour la vente au détail.

Ainsi, les données obtenues à partir de ces conjectures ont permis d'estimer la rentabilité annuelle d'un élevage en fonction des systèmes par l'approche comptable (Keiser, 2004). Les marges brute et nette ont été déterminées par les formules suivantes :

Marge brute $=$

produit brut d'exploitation - charges opérationnelles totales

Marge nette $=$ recettes totales - dépenses totales ou

Marge nette $=$ marge brute - charges fixes

Coût de production d'un porc charcutier $=\frac{\text { total des charges }}{\text { nb. de porcs produits }}$

\section{RESULTATS}

\section{Profil socioéconomique et motivation des éleveurs}

Lélevage porcin est une activité qui occupe à la fois les hommes et les femmes (50,2 \% d'hommes et 49,8\% de femmes). Il est pratiqué par des éleveurs issus d'une dizaine d'ethnies avec une prédominance de Diolas (41,3\%), de Mancagnes (36,5\%) et de Balantes (10,8\%). Les propriétaires de ces élevages étaient pour la plupart chrétiens $(95,98 \%)$, mariés $65,3 \%$ et responsables de famille nombreuse $(9,4$ $\pm 5,2$ personnes par ménage). Ils étaient en majorité instruits : $31,6 \%$ avaient le niveau d'étude du primaire et $36,2 \%$ celui du secondaire. Les éleveurs s'inscrivaient dans divers secteurs d'activités, notamment l'agriculture $(24,8 \%)$, le commerce $(16,9 \%)$ et autres $(9 \%)$. Dans la région de Sédhiou, l'association élevage-agriculture était dominante $(45,4 \%)$, alors qu'à Ziguinchor, où la plupart des éleveurs étaient généralement instruits $(79,4 \%)$, ceux qui ne pratiquaient que l'élevage $(30,5 \%)$ étaient majoritaires. La plupart $(78,2 \%)$ des éleveurs de ces régions du Sud avaient en général plus de dix ans d'expérience dans la conduite des porcs malgré leur manque de formation sur les techniques d'élevage (83,5\%) (tableau I). Aussi, ils utilisaient la propriété familiale $(92,4 \%)$ avec des porcs généralement acquis par achat de paires de porcelets sevrés $(82,7 \%)$ ou reçus comme don $(19,8 \%$ ). La main-d'œuvre au niveau des élevages porcins était, dans la plupart des cas, familiale $(97,2 \%)$. Dans les trois régions, les éleveurs de porcs avaient démarré leur activité sur leur propre initiative $(76,8 \%)$. Ils pratiquaient d'ailleurs cet élevage à la fois pour la génération de revenus $(95,4 \%)$ et l'autoconsommation $(81,1 \%)$.

\section{Systèmes d'élevage de porcs}

Trois systèmes d'élevage ont été identifiés : le système de production traditionnel, le système semi-intensif et le système intensif. Ces trois systèmes ont été définis selon des critères comme le type d'habitat, l'alimentation, la conduite et le suivi des activités d'élevage. Dans le système traditionnel l'habitat était en banco ou en bois, ou en matériau de fortune, les porcs étaient généralement de race locale et laissés en divagation, sans ou avec apport de complément alimentaire (restes de cuisine en général), et sans suivi sanitaire de la part de l'éleveur. Dans le système intensif, l'habitat était en matériau définitif. La stabulation des porcs, majoritairement des races améliorées, était complète avec un plan correct de suivi sanitaire et l'alimentation était constituée de provendes industrielles mélangées ou non avec des sous-produits agroalimentaires. Dans le système semi-intensif, à mi-chemin entre les deux systèmes précédents, l'habitat était en matériau semi-définitif ou en banco amélioré, les porcs étaient

\section{Tableau I}

Profil socioprofessionnel et motivations des éleveurs de porcs dans la Casamance naturelle (Sénégal) (\%)

\begin{tabular}{|c|c|c|c|c|}
\hline Paramètres & Kolda & Sédhiou & Ziguinchor & Moyenne \\
\hline \multicolumn{5}{|l|}{ Sexe } \\
\hline Masculin & 51,6 & 48,9 & 50 & 50,1 \\
\hline Féminin & 48,4 & 51,1 & 50 & 49,9 \\
\hline \multicolumn{5}{|l|}{ Ethnie } \\
\hline Manjacque & 4,4 & 6,9 & 7,2 & 6,3 \\
\hline Mancagne & 50 & 50,6 & 18,8 & 36,5 \\
\hline Balante & 13,3 & 24,1 & 0,7 & 10,8 \\
\hline Diola & 25,6 & 11,5 & 70,3 & 41,3 \\
\hline Massonké & 1,1 & 6,9 & 0 & 2,2 \\
\hline Autre & 5,6 & 0 & 2,9 & 2,9 \\
\hline \multicolumn{5}{|l|}{ Statut marital } \\
\hline Marié & 64,4 & 62,5 & 67,6 & 65,3 \\
\hline Célibataire & 10 & 10,2 & 21,1 & 15 \\
\hline Divorcé & 3,3 & 4,5 & 1,4 & 2,8 \\
\hline Veuf & 20 & 22,7 & 7,7 & 15,3 \\
\hline Religieux & 2,2 & 0 & 2,1 & 1,6 \\
\hline \multicolumn{5}{|l|}{ Niveau d'études } \\
\hline Aucun & 23 & 37,5 & 20,6 & 25,9 \\
\hline Primaire & 29,7 & 27,3 & 35,5 & 31,6 \\
\hline Secondaire & 38,5 & 29,5 & 39 & 36,2 \\
\hline Supérieur & 8,8 & 5,7 & 5 & 6,2 \\
\hline \multicolumn{5}{|l|}{ Principale activité } \\
\hline Fonction publique & 8,9 & 6,8 & 9,2 & 8,5 \\
\hline Professionnel privé & 27,8 & 12,5 & 17,2 & 18,8 \\
\hline Commerçant & 15,6 & 17 & 17,7 & 16,9 \\
\hline Agriculteur & 13,3 & 45,4 & 19,1 & 24,8 \\
\hline Eleveur & 20 & 10,2 & 30,5 & 21,9 \\
\hline Autre & 14,4 & 8 & 6,4 & 9,1 \\
\hline \multicolumn{5}{|l|}{ Motivation } \\
\hline Conseil d'autrui & 4,4 & 3,4 & 6,2 & 4,9 \\
\hline Propre initiative & 70,3 & 80,7 & 78,5 & 76,8 \\
\hline Imitation & 2,2 & 3,4 & 3,5 & 3,1 \\
\hline Héritage familial & 27,5 & 14,8 & 13,9 & 18 \\
\hline \multicolumn{5}{|l|}{ Objectif de production } \\
\hline Autoconsommation & 97,8 & 75 & 74,3 & 81,1 \\
\hline Source de revenu & 97,8 & 98,9 & 91,7 & 95,4 \\
\hline Autre & 2,2 & 3,4 & 14,6 & 7,7 \\
\hline \multicolumn{5}{|c|}{ Formation en porciculture } \\
\hline Oui & 0 & 21,6 & 23,8 & 16,5 \\
\hline Non & 100 & 78,4 & 76,2 & 83,5 \\
\hline \multicolumn{5}{|c|}{ Expérience en élevage porcin } \\
\hline$<5$ ans & 7,7 & 10,2 & 21,8 & 14,6 \\
\hline $5-10$ ans & 7,7 & 6,8 & 7 & 7,2 \\
\hline$>10$ ans & 84,6 & 82,9 & 71,1 & 78,2 \\
\hline
\end{tabular}

de races mixtes (locale, métisse, améliorée), ils bénéficiaient d'un suivi sanitaire, leur stabulation était complète et l'alimentation était plus ou moins équilibrée, composée d'un mélange de deux à trois matières premières et de déchets agricoles. Nos résultats ont montré que le système d'élevage traditionnel était majoritaire avec 85,5\% des élevages enquêtés indépendamment de la région (figure 2). 


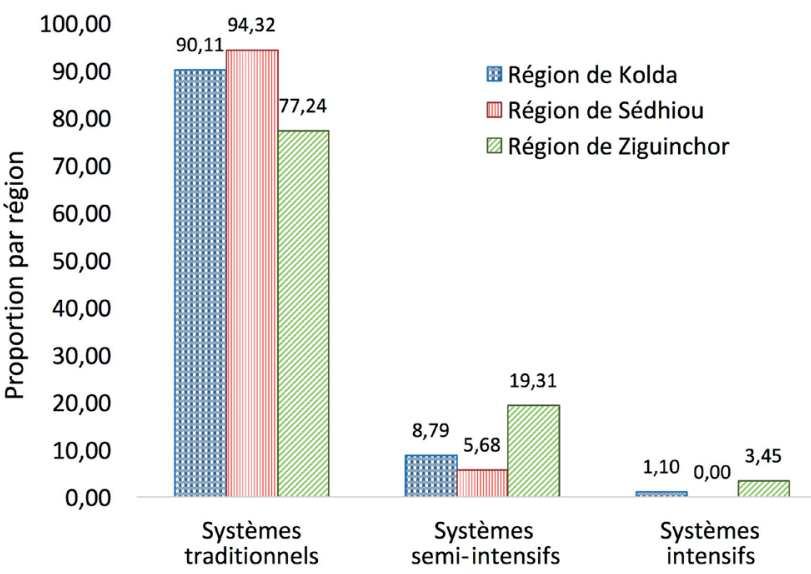

Figure 2: répartition des systèmes d'élevage dans les régions de la Casamance (Sénégal).

\section{Types de production, races exploitées, taille des troupeaux}

Les élevages porcins visités n'étaient pas spécialisés. Ils étaient à la fois de type naisseur et engraisseur (95,3\%), et exploitaient principalement la race locale $(90,4 \%)$ qui reste très caractéristique des systèmes traditionnels $(94,2 \%)$. Les races améliorées et métissées étaient surtout rencontrées dans le système intensif (respectivement 83,3\% et 66,7 \%) par rapport au système semi-intensif (respectivement $26,8 \%$ et $46,3 \%$ ). Les proportions observées étaient quasi identiques entre les régions.

La taille et la composition des troupeaux des élevages enquêtés sont présentées dans le tableau II. En moyenne, chaque exploitation comptait $19,2 \pm 20$ porcs, avec un minimum de 1 et un maximum de 180 porcs. Les porcs reproducteurs représentaient $31 \%$ du troupeau (dont $15 \%$ de mâles et $16 \%$ de femelles). Les grands effectifs de porcs étaient rencontrés à Kolda $(28,2 \pm 20,8)$. En fonction des systèmes d'élevage, la taille moyenne du troupeau du système intensif $(28,2 \pm 15,6$ porcs avec $29 \%$ de reproducteurs) a été plus importante que celles du système traditionnel $(19,4 \pm 21$ porcs avec $31 \%$ de reproducteurs) et du système semiintensif (16,9 $\pm 12,7$ porcs avec $24 \%$ de reproducteurs).

\section{Porcheries et matériel d'élevage}

Parmi les élevages enquêtés, seuls 3,4\% ne disposaient pas d'habitats pour porcs. Les différents types de porcheries rencontrés étaient des abris traditionnels $(51,1 \%)$, des abris semi-modernes (14,5\%), des abris modernes $(6 \%)$ et des abris de fortune $(28,4 \%)$. Ces derniers consistaient en de petites porcheries très rudimentaires, avec des toitures en paille ou en tôles de zinc rouillé. Les parois latérales étaient principalement en bois. Tous avaient un plancher en terre battue. Les porcheries traditionnelles correspondaient à une version améliorée des précédents ; les tôles de récupération étaient les plus utilisées comme toitures, mais

\section{Tableau II}

Taille et composition ( $n b$. de têtes) du troupeau des élevages porcins dans les trois régions de la Casamance naturelle (Sénégal)

\begin{tabular}{lccc} 
& Kolda & Sedhiou & Ziguinchor \\
\hline Taille par exploitation & $28,2 \pm 20,8$ & $18,2 \pm 24,2$ & $14,3 \pm 14,2$ \\
Porcelets non sevrés & $12,0 \pm 11,2$ & $7,6 \pm 9,3$ & $5,6 \pm 7,8$ \\
Porcs en croissance & $11,7 \pm 9,8$ & $7,1 \pm 7,1$ & $6,1 \pm 5,4$ \\
Porcs reproducteurs & $6,4 \pm 5,3$ & $5,7 \pm 9,4$ & $4,7 \pm 4,6$
\end{tabular}

aussi les pailles ou quelques fois les bâches. Les murs étaient principalement en bois ou en briques d'un côté, et en bois de l'autre. Les porcheries semi-modernes avaient des toitures en tôles ou en tuiles. Les parois latérales étaient construites en briques et le plus souvent cimentées. Le plancher était cimenté en partie ou en totalité. Les porcheries modernes étaient compartimentées en fonction du stade physiologique des animaux, avec une toiture en tôles ou en tuiles, des parois latérales en briques bien cimentées, de même que le plancher.

Ces porcheries étaient équipées de mangeoires et d'abreuvoirs très divers (33\% de bois creusé, $28 \%$ de bois creusé et de bidons coupés, $25,2 \%$ d'ustensiles de cuisine reformés), et d'autres types de matériel $(14,2 \%)$. Les mangeoires ou abreuvoirs en bois étaient les plus utilisés $(63,1 \%)$. Ils avaient l'avantage de durer très longtemps mais ils étaient chers par rapport aux fûts fendus et ustensiles de cuisine réformés. Ils restaient majoritaires (65,7\% des exploitations) dans le système traditionnel. Ils occupaient la deuxième place, après les mangeoires et abreuvoirs cimentés et bétonnés, dans les autres systèmes d'élevage (43,2 \% en élevage semi-intensif et $25 \%$ en élevage intensif). Les autres matériels rencontrés étaient principalement des seaux $(70,4 \%)$ et des balais $(45,7 \%)$. Les pelles, brouettes, râteaux et autres matériels étaient davantage destinés à l'agriculture qu’à l'élevage.

\section{Conduite de l'alimentation}

L'alimentation des porcs était surtout à base de déchets alimentaires et de verdures. Les aliments utilisés étaient formulés dans la plupart des exploitations (97\%) par les éleveurs eux-mêmes et seuls 5,3\% d'entre eux les pesaient avant de les distribuer aux porcs. La distribution des aliments avait lieu majoritairement $(64,7 \%)$ deux fois par jour, notamment le matin et le soir. Trois types d'aliments ont été répertoriés en fonction de leur nature. Le type 1 était constitué des restes de cuisine et autres résidus agricoles associés à de la verdure (petites plantes et herbes). Le type 2, le plus utilisé (76,1\%), comprenait un mélange de deux ou plusieurs matières premières auxquelles l'éleveur ajoutait des déchets alimentaires récupérés auprès des ménages et des restaurants, de la verdure et d'autres aliments. Enfin, l'aliment complet (ou provende) utilisé seul ou mélangé à d'autres matières premières (son de riz, farine de récupération des meuneries, etc.) caractérisait le type 3. D'après les déclarations des éleveurs, les matières alimentaires les plus utilisées dans les rations étaient les déchets alimentaires (90,1\% des cas), les mangues $(56 \%)$, les noix palmistes (54\%), les verdures et légumes (53\%), et autres (figure 3).

En fonction des systèmes d'élevage, la nature des aliments pouvait différer. L'aliment industriel (provende porc) était plus souvent employé

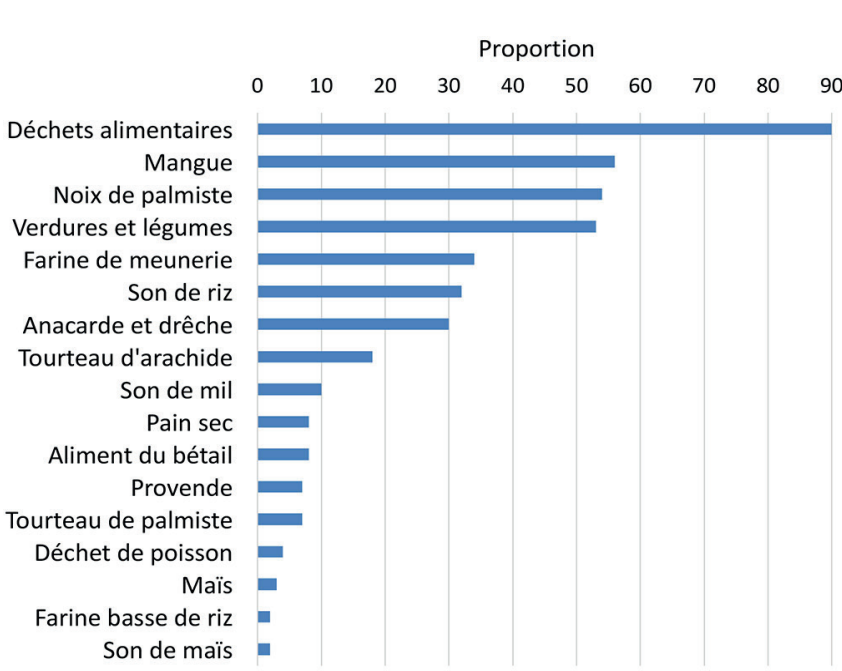

Figure 3: fréquence d'utilisation des ressources alimentaires dans l'alimentation des porcs en Casamance (Sénégal). 
dans les systèmes intensif $(83,3 \%)$ et semi-intensif $(19,5 \%)$ que dans le système traditionnel $(3,9 \%)$. Son acquisition s'effectuait principalement auprès d'un provendier $(77,3 \%)$ ou était offerte $(13,6 \%)$ par le Projet d'appui à la sécurité alimentaire et à l'élevage (Pasael). Toutefois, cet aliment dont le prix moyen variait de 7000 à 12000 FCFA par sac de $50 \mathrm{~kg}$ (soit 140-240 FCFA $/ \mathrm{kg}$ ) était généralement mélangé par les éleveurs avec d'autres intrants moins coûteux pour des raisons économiques.

Selon 90,2 \% des éleveurs, trouver des aliments pour porcs n’était pas aisé. Les entraves citées étaient surtout l'indisponibilité de certaines matières premières $(70,8 \%)$, leur cherté $(11 \%)$ ou les deux à la fois $(12,8 \%)$. Mais, le problème d'accessibilité aux aliments complets pour porc était une contrainte commune à tous les éleveurs, indépendamment du système d'élevage (100 \% des éleveurs du système intensif, 86,8 \% de ceux du système semi-intensif et $90,5 \%$ de ceux du système traditionnel).

\section{Conduite de la reproduction et productivité}

Ces paramètres concernaient le mode de reproduction, l'âge de mise à la reproduction, la taille de la portée et le sevrage. La reproduction se faisait par monte naturelle dans toutes les exploitations porcines et de manière non contrôlée $(98,1 \%$ ). L'âge moyen de mise à la reproduction était de 7,6 $\pm 1,8$ mois, la taille moyenne par portée était de 7,4 $\pm 2,2$ porcelets et les taux de mortalités étaient de $16,5 \%$ et $12,1 \%$, respectivement avant et après le sevrage (tableau III).

Ces résultats étaient cependant sujets à des variations selon le système d'élevage. Dans le système traditionnel, l'âge moyen de la mise à la reproduction était de 7,5 $\pm 1,7$ mois. En réalité les éleveurs ne contrôlaient pas ce paramètre puisque la reproduction se faisait le plus souvent sans leur intervention en raison de la divagation. Ce paramètre était de 7,7 \pm 1,6 mois dans le système semi-intensif et de 7,6 $\pm 2,5$ dans le système intensif. La taille de la portée à la naissance était plus élevée dans le système intensif $(10,4 \pm 3,9)$ que dans les systèmes semi-intensif $(7,5 \pm 2,1)$ et traditionnel $(7,3 \pm 2)$. Les mortalités respectivement avant et après sevrage étaient plus élevées dans les élevages semi-intensifs $(28,8 \%$ et $14,1 \%)$ que dans les élevages traditionnels $(22,3 \%$ et $12,2 \%)$ et intensifs $(4,5 \%$ et $0 \%)$.

Le sevrage se faisait majoritairement par la truie qui se débarrassait de ses petits entre 3 et 6 mois dans les systèmes traditionnel (dans $90 \%$ des cas) et semi-intensif (70,3\%). Il était contrôlé par les éleveurs dans le système intensif et pratiqué à 1,5 mois ( $40 \%$ de cas) ou entre 2 et 3 mois (60\% des cas).

Certaines pratiques visaient à améliorer la conformation des porcs et leur identification. Ainsi, la castration était pratiquée dans $96,8 \%$ des élevages enquêtés, principalement par les éleveurs $(82,5 \%)$. Elle était réalisée chez les porcs à un âge variable entre 3 et 5 mois (31,8\%), 6 et 12 mois $(27 \%)$ ou quand l'animal devenait turbulent $(24 \%)$. La pratique de la caudectomie était peu répandue (14,6\%), les éleveurs préférant le marquage à l'oreille (74,5\%). Utilisé en guise d'identification, ce marquage, réalisé par les éleveurs eux-mêmes (96,9\%), intervenait le plus souvent après la naissance des porcelets (à moins d'un mois d'âge).

\section{Gestion sanitaire}

L'offre de services vétérinaires était faible et la prise en charge sanitaire des élevages porcins demeurait en grande partie traditionnelle avec des pratiques médicales parfois douteuses. Le nettoyage des porcheries était effectué dans $77,4 \%$ des élevages sans désinfectant (76\%). La vaccination était peu pratiquée $(14,7 \%)$ et les éleveurs ignoraient les maladies contre lesquelles elle est réalisée $(53,1 \%)$ de même que les produits utilisés $(50 \%)$. Dans 33,3\% des exploitations, les éleveurs ont affirmé pratiquer le déparasitage. Cependant, les produits le plus souvent employés comme le sel $(4,9 \%)$, le Crésyl $(1,2 \%)$, et autres produits traditionnels $(34,6 \%)$ ou inconnus des éleveurs $(25,9 \%$, les urines par exemple) n'étaient pas reconnus comme antiparasitaires en médecine vétérinaire. En cas de maladie, soit les éleveurs n'intervenaient pas (33,9\%), soit ils traitaient les porcs avec des produits traditionnels (35\%) ou des médicaments vétérinaires $(21,2 \%)$. Une faible proportion d'éleveurs $(9,8 \%)$ associaient ces deux types de traitements. Dans ces élevages, le suivi sanitaire était du ressort de l'éleveur (81,1 \%), à l'exception de $4 \%$ d'entre eux qui recouraient aux services d'un vétérinaire.

\section{Mode d'exploitation des porcs}

Les porcs étaient exploités surtout pour l'autoconsommation et la quête de revenus à travers la vente. Concernant l'autoconsommation, 87,4\% des éleveurs prélevaient en moyenne $3,9 \pm 2,7$ porcs par an, ce qui représentait 20,3\% du troupeau. Ce prélèvement était souvent effectué à l'occasion des fêtes et cérémonies religieuses $(93,9 \%)$, mais également pour les besoins de consommation ordinaire de la famille $(68,9 \%)$.

Par rapport à la vente de porcs, notre étude a révélé l'existence de deux systèmes de commercialisation : la vente sur pieds et la vente en détail. Le premier, le plus répandu, était pratiqué par 82,2 \% des éleveurs. Le second était très rarement exercé seul $(1 \%)$ et associait fréquemment la vente sur pieds $(16,9 \%)$. Pourtant, il n'existait pas dans les marchés ou louma des places destinées à la vente de porcs. Ainsi, les animaux étaient vendus principalement à domicile $(96,5 \%)$ sur décision de l'éleveur et les prix étaient débattus pour en estimer la valeur (95\%). Le prix moyen d'un porc sur pieds et le nombre de porcs vendus par an étaient variables d'un système d'élevage à l'autre. Les plus grandes ventes (40 porcs) et les meilleurs prix (43250 FCFA/porc, 1433 FCFA/kg de poids

\section{Tableau III}

Performances moyennes de reproduction dans les systèmes d'élevage porcin de la Casamance naturelle (Sénégal)

\begin{tabular}{|c|c|c|c|}
\hline Paramètres de reproduction & Système traditionnel & Système semi-intensif & Système intensif \\
\hline Age mise à la reproduction (mois) & $7,5 \pm 1,7$ & $7,7 \pm 1,6$ & $7,6 \pm 2,5$ \\
\hline Age à la $1^{\mathrm{re}}$ mise-bas (mois) & $13,5 \pm 5,6$ & $13,8 \pm 7$ & $12 \pm 2,7$ \\
\hline $\mathrm{Nb}$. de gestations / truie/ an & $1,9 \pm 0,3$ & $1,8 \pm 0,4$ & $1,7 \pm 0,5$ \\
\hline $\mathrm{Nb}$. de portées / truie / an & $1,9 \pm 0,3$ & $1,9 \pm 0,3$ & $1,7 \pm 0,5$ \\
\hline Avortements/an & $0,4 \pm 0,9$ & $0,5 \pm 1$ & $2 \pm 3,4$ \\
\hline Taille moyenne / portée & $7,3 \pm 2,1$ & $7,5 \pm 2,1$ & $10,4 \pm 3,9$ \\
\hline Mort-nés & $0,6 \pm 0,9$ & $0,7 \pm 1,6$ & $0,2 \pm 0,4$ \\
\hline Nés vifs & $6,7 \pm 2$ & $6,6 \pm 2,8$ & $10,2 \pm 4,1$ \\
\hline Mortalités présevrage (\%) & 22,3 & 28,8 & 4,5 \\
\hline Taille de la portée au sevrage & $5,7 \pm 2,2$ & $5,4 \pm 2,5$ & $8,4 \pm 5,4$ \\
\hline Mortalités postsevrage (\%) & 12,1 & 14,1 & 0 \\
\hline
\end{tabular}


vif, 2000 FCFA $/ \mathrm{kg}$ de viande) ont été rencontrés dans le système intensif. Les ventes moins importantes ont été enregistrées dans le système traditionnel ( 9 porcs vendus 33382 FCFA chacun) et le système semiintensif (10 porcs vendus 38125 FCFA chacun, $1500 \mathrm{FCFA} / \mathrm{kg}$ de poids vif, $1650 \mathrm{FCFA} / \mathrm{kg}$ de viande). La vente au poids vif ou sous forme de viande était l'apanage des systèmes intensif et semi-intensif. Ces modes de vente étaient absents du système traditionnel où prédominait la vente basée sur l'estimation. Les grandes périodes de vente de porcs étaient pendant les fêtes, les cérémonies chrétiennes (48,5\%), la saison sèche $(25,9 \%)$, voire à tout moment pour certains éleveurs $(21,4 \%)$.

Les déjections des porcs ne faisaient pas l'objet de transaction. Elles étaient principalement utilisées comme fumier en épandage (70\%) dans l'activité agricole des éleveurs, et accessoirement offertes aux voisins et amis, ou jetées dans la nature en l'absence de demande.

Les porcs étaient principalement vendus pour des besoins pécuniaires $(90,9 \%)$ à des Guinéens $(58,9 \%)$ et aux ménages casamançais $(57,2 \%)$. Par ordre décroissant, les Bissau-Guinéens faisaient davantage leurs achats dans la région de Kolda $(93,2 \%)$ et de Sédhiou $(83,9 \%)$ que dans celle de Ziguinchor (20\%) pourtant plus proche de leur pays. Par contre, les salariés locaux étaient les clients majoritaires $(75,4 \%)$ dans la région de Ziguinchor (tableau IV). La Gambie et la région de Dakar représentaient des destinations négligeables pour la vente de porcs.

L'exploitation porcine était une activité informelle en l'absence d'outils de suivi des performances et d'enregistrement des données technicoéconomiques (97\% des élevages). Toutefois, la commercialisation des produits selon les déclarations des éleveurs était rentable. Ainsi, 67,5\% d'entre eux ont qualifié leur activité d'assez à très rentable. Peu d'éleveurs des systèmes traditionnel $(16,9 \%)$ et semi-intensif $(14,3 \%)$ ont considéré leur élevage comme une activité non rentable.

\section{Coûts de production et rentabilité}

Les résultats économiques (tableau V) concernaient les élevages naisseurs engraisseurs. Ils étaient préliminaires et calculés sur la base des estimations et des déclarations des éleveurs. Il ressort des analyses que les charges alimentaires constituaient le facteur déterminant de la rentabilité des élevages porcins. Elles représentaient en moyenne 65,3\% des dépenses et étaient plus élevées dans le système moderne $(78,6 \%)$ que dans les systèmes semi-intensif $(47,6 \%)$ et traditionnel $(69,7 \%)$. La même tendance a été observée pour la marge brute annuelle, la plus grande valeur de 785109 FCFA ayant été enregistrée dans le système intensif.
Par ailleurs, les résultats ont montré que les coûts moyens de production d'un porc charcutier étaient de 25816 FCFA dans le système traditionnel, 53352 FCFA dans le système semi-intensif et 83407 FCFA dans le système intensif, respectivement pour des porcs de 40,80 et $90 \mathrm{~kg}$. En considérant le prix de vente d'un porc et les différentes méthodes de vente selon le système d'élevage, un bénéfice net de 7566 FCFA était perçu par porc engraissé et vendu dans le système traditionnel. Ce bénéfice était légèrement supérieur à celui estimé dans les systèmes semiintensif (5435 FCFA/porc) et intensif (6061 FCFA/porc).

\section{DISCUSSION}

\section{Profil socioéconomique des éleveurs}

La proportion des hommes propriétaires de porcs $(50,1 \%)$ était quasi équivalente à celle des femmes (49,9\%). Ce résultat semble unique, car en Afrique de l'Ouest et du Centre, l'élevage porcin est dominé soit par des hommes (Ayssiwede et al., 2008 ; Youssao et al., 2008 ; Ndebi et al., 2009 ; Umutoni, 2012) soit par des femmes (FAO, 2012a ; FAO, 2012b ; Missohou et al., 2001 ; Sambou, 2008). Cette différence peut s'expliquer par le système d'élevage. En effet, dans les études où les hommes prédominaient, les élevages avaient un profil plus ou moins intensif ou semi-intensif. En revanche, les femmes prédominaient dans les études sur les élevages autrefois pratiqués pour la subsistance familiale (consommation, dons) et qui associaient l'élevage de porcs au recyclage des déchets organiques des décharges (FAO, 2012a ; Sambou, 2008). Sur un intervalle de 15 ans, depuis les travaux de Missohou et al. (2001) dans la basse Casamance, les hommes se sont davantage intéressés à l'élevage de porc. Le chômage ou la recherche de diversification de sources de revenus expliqueraient cette nouvelle tendance. En effet, sur la répartition des activités entre l'homme et la femme, Mbetid-Bessane et Mohamed (2003) ont conclu que la répartition selon le genre des activités n'est pas irréversible. L'homme peut changer de comportement dès lors qu'une activité traditionnellement exercée par les femmes devient assez rémunératrice pour constituer une activité de rente. La culture de riz en Casamance, par exemple, était une culture pratiquée par les femmes puisqu'elles s'occupaient de la nourriture et le riz ne servait qu'à l'autoconsommation. Dès lors qu'il est devenu assez rémunérateur, les hommes s'y sont intéressés. Ce changement de comportement dans une quelconque activité nous a semblé assez pertinent et expliquait comment la recherche de revenus était le principal objectif de production commun à 95,4\% des éleveurs enquêtés. Cela était d'autant plus vrai que la plupart $(76,8 \%)$ avaient entrepris cette activité sur leur propre initiative.

\section{Tableau IV}

Raisons de l'exploitation commerciale des porcs et principaux clients dans la Casamance naturelle (Sénégal)

\begin{tabular}{|c|c|c|c|c|c|}
\hline Paramètres & & $\begin{array}{l}\text { Kolda } \\
(\%)\end{array}$ & $\begin{array}{c}\text { Sédhiou } \\
(\%)\end{array}$ & $\begin{array}{c}\text { Ziguinchor } \\
(\%)\end{array}$ & $\begin{array}{c}\text { Moyenne } \\
(\%)\end{array}$ \\
\hline \multirow[t]{5}{*}{ Raisons de vente } & Besoin d'argent & 90,9 & 91,2 & 90,8 & 90,9 \\
\hline & Réforme & 5,7 & 3,7 & 10,9 & 7,3 \\
\hline & Déstockage & 38,6 & 28,7 & 26 & 30,7 \\
\hline & Crainte des épidémies & 9,1 & 27,5 & 5,9 & 12,9 \\
\hline & Crainte de vol & 2,3 & 0 & 2,5 & 1,7 \\
\hline \multirow[t]{6}{*}{ Clients } & Commerçant revendeur & 6,8 & 16 & 14,6 & 12,7 \\
\hline & Charcuterie, restaurant, hôtel & 3,4 & 1,2 & 25,4 & 12,4 \\
\hline & Salarié local et particulier & 43,2 & 43,2 & 75,4 & 57,2 \\
\hline & Expatrié & 7,9 & 3,7 & 8,5 & 7 \\
\hline & Autre éleveur ou villageois & 18,2 & 17,3 & 27,7 & 22,1 \\
\hline & Bissau-Guinéen & 93,2 & 83,9 & 20 & 58,9 \\
\hline
\end{tabular}




\section{Tableau V}

Résultats annuels d'une exploitation porcine selon le système d'élevage dans la Casamance naturelle (Sénégal) (FCFA)*

\begin{tabular}{lrrr}
\hline & \multicolumn{3}{c}{ Système d'élevage } \\
\cline { 2 - 4 } & Traditionnel & Semi-intensif & Intensif \\
\hline $\begin{array}{l}\text { Dépenses - charges fixes } \\
\text { Main-d'œuvre }\end{array}$ & 0 & 184500 & 318000 \\
Amortissement annuel & 43206 & 45636 & 224655 \\
Dépenses - charges variables & & & \\
$\quad$ Alimentation & 161841 & 253748 & 2623200 \\
$\quad$ Nettoyage & 0 & 18104 & 28437 \\
$\quad$ Frais vétérinaires & 12075 & 15375 & 48000 \\
$\quad$ Déplacement & 15218 & 16160 & 94000 \\
Total des dépenses & 232340 & 533524 & 3336292 \\
& & & \\
Recettes - vente d'animaux & 300438 & 587875 & 3578746 \\
Marge brute annuelle & 111304 & 284487 & 785109 \\
Marge nette annuelle & 68098 & 54351 & 242454 \\
Coût de production / porc & 25816 & 53352 & 83407 \\
Charges alimentaires (\%) & 69,7 & 47,56 & 78,6 \\
Bénéfice net / porc & 7566 & 5435 & 6061
\end{tabular}

* 1000 francs $\mathrm{CFA}=1,52$ euro

En outre, les résultats montrent le caractère multiethnique des éleveurs avec une prépondérance de l'ethnie Diola (41,3\%). Ces résultats sont en accord avec ceux de Missohou et al. (2001), Fall (2008), et Secka (2011), mais ils ne concordent pas avec ceux de Buldgen et al. (1994), et Sambou (2008). Ces derniers rapportent que $87 \%$ des éleveurs de porcs dans le bassin arachidier appartiennent à l'ethnie Sérère et $86,7 \%$ des éleveurs de porcs autour de la décharge de Mbeubeuss (Dakar) sont de l'ethnie Manjaque. Nous estimons que cette différence est due à la répartition historiquement inégale des ethnies entre les différentes régions du pays.

Par ailleurs, près de $25 \%$ des personnes interrogées associaient l'élevage de porcs à l'agriculture. Nos résultats sont similaires à ceux obtenus en zone rurale par Ndebi et al. (2009) au Cameroun, et Mopaté Logténé et al. (2010) au Tchad. Comme observé par le CSE (2007), l'élevage est pratiqué par des agriculteurs chez qui les cultures pluviales constituent une source de revenus supplémentaires et représentent de 10 à $50 \%$ du revenu brut au Sénégal. Cependant nos résultats diffèrent de ceux d'auteurs ayant travaillé sur des élevages en zones périurbaines de villes africaines comme Dakar, Cotonou, Abomey-Calavi et Bobo-Dioulasso (Sambou, 2008 ; Youssao et al., 2008 ; Doumana, 2011 ; Umutoni, 2012). Dans ces études, la grande partie des éleveurs est constituée respectivement par des fonctionnaires $(50 \%)$, ceux qui font de l'élevage uniquement $(80 \%)$, des ménagères $(53,3 \%)$, et des artisans $(44 \%)$. Enfin, nos résultats ont montré que la plupart des éleveurs de porcs de la Casamance $(78,2 \%)$ ont plus de dix ans d'expérience dans cette activité, et peu d'éleveurs $(14,6 \%)$ l'ont adoptée il y a moins de cinq ans. La nonimplication de nouveaux éleveurs, contrairement aux résultats de Tra Bi Tra (2009) en Côte d'Ivoire (46,2 \%) et Ndebi et al. (2009) au Cameroun $(40,9 \%)$, serait due au découragement. Il fait suite aux différentes contraintes, en particulier la cherté, l'accès à l'aliment et à l'espace, la faiblesse des services techniques, les barrières socioculturelles et religieuses, et les pathologies (Ossebi et al., 2018). Parmi ces pathologies, la peste porcine africaine, avec une séroprévalence entre 30 et $32 \%$ en Casamance (Seck, 2007 ; Akpaki et al., données non publiées), constitue un véritable fléau pour cet élevage de par son caractère endémique.

\section{Caractéristiques fonctionnelles des élevages}

\section{Systèmes d'élevage et taille des troupeaux}

Au sud du Sénégal, le système traditionnel d'élevage de porcs reste de loin prédominant $(85,5 \%)$, et la race locale est la plus exploitée comparée aux races métisses et exotiques. Ces résultats corroborent ceux de Buldgen et al. (1994) dans le bassin arachidier du Sénégal, de Missohou et al. (2001) en basse Casamance, et de Secka (2011) en Casamance et en Gambie. En Afrique centrale et de l'Ouest plusieurs auteurs (Ayssiwede et al., 2008 ; Tra Bi Tra, 2009 ; Ndebi et al., 2009 ; Umutoni, 2012 ; Agbokounou et al., 2016b) rapportent que le système traditionnel est surtout rencontré en milieu paysan et dans les forêts.

Par ailleurs, la taille moyenne des exploitations visitées (19,2 porcs) était proche de celle rapportée par Sambou (2008) à Dakar (20,8 porcs), mais faible comparée à celle relevée par Ayssiwede et al. (2008) au Bénin $(40,65$ porcs). Cette différence peut s'expliquer par le différentiel du niveau de développement des pratiques de la porciculture, de pesanteurs socioculturelles et surtout du marché. Au Bénin, il y a beaucoup de chrétiens et d'animistes (Houndonougbo et al., 2012) et la demande en viande de porc est beaucoup plus élevée qu'au Sénégal. Ce résultat est supérieur à celui obtenu par Le Goulven et al. (1999) au Vietnam (deux porcs). De même, il est plus important que ceux de Buldgen et al. (1994) et Missohou et al. (2001) qui ont trouvé respectivement deux porcs par exploitation dans le bassin arachidier du Sénégal et $11 \pm 9,1$ porcs en basse Casamance. Au Sénégal, l'évolution des effectifs dans le temps (croissance de 3,2 \%) et la période de l'année peuvent justifier cet écart. De plus, en saison des pluies, la stabulation obligatoire associée à une disette d'aliments ne permet pas aux éleveurs de garder des effectifs élevés de porcs. Les pesanteurs socioculturelles et religieuses comme le vol, l'empoisonnement (Lalèyê, 2007) et la cohabitation des chrétiens et des musulmans contribuent également à la limitation des effectifs de porcs par les éleveurs.

\section{Porcheries et matériel d'élevage}

Les habitats étaient principalement des porcheries traditionnelles améliorées $(51,1 \%)$ et des abris de fortune $(28,4 \%)$. Nos résultats sont similaires à ceux rapportés en Afrique en général par Mopaté Logténé et al. (2010), Ndebi et al. (2009), Secka (2011), et Agbokounou et al. (2016b). Cependant, ils sont différents d'autres travaux conduits au Sénégal (Sambou, 2008 ; Bassene, 2010 ; Doumana, 2011), au Bénin (Ayssiwede et al., 2008) et au Burkina Faso (Umutoni, 2012) qui notent que les bâtiments améliorés ou semi-modernes sont majoritaires. Cette différence s'explique par la localisation des élevages enquêtés. Les élevages des zones périurbaines des principaux centres économiques sont le plus souvent améliorés. Les propriétaires de terres périurbaines ont souvent d'autres sources de revenus et ont accès au système financier leur permettant dans l'ensemble de réaliser les investissements nécessaires (Filmer et Fox, 2014). En revanche, pour les agroéleveurs, majoritaires dans notre étude, les pratiques consistent à thésauriser l'excédent de trésorerie afin de constituer un capital nécessaire pour réaliser les investissements (Mbetid-Bessane et al., 2003). Les caractéristiques du matériel d'élevage dans notre étude étaient similaires à celles rapportées par Abdallah-Nguertoum (1997), et Agbokounou et al. (2016b).

\section{Conduite de l'alimentation}

Les résultats ont montré que les éleveurs préparaient eux-mêmes les aliments et ne tenaient pas compte de l'équilibre nutritionnel de la ration. Ces résultats concordent avec ceux de Buldgen et al. (1994), et d'Agbokounou et al. (2016b) qui rapportent que les restes de repas, de cuisine, le mil, le riz, les épluchures de manioc ou de patate douce, la feuille de baobab, le pulpe de tomate ou la feuille de chou servent de base dans les régimes alimentaires. La valorisation rationnelle des ressources alimentaires locales peut constituer une voie alternative pour améliorer l'alimentation des porcs. Toutefois, les résultats obtenus en Côte d'Ivoire par Tra Bi Tra (2009) et au Bénin par Ayssiwede et al. (2008) diffèrent des 
nôtres. Ces auteurs stipulent que plus de $90 \%$ des éleveurs recourent à la provende, surtout en milieu urbain et périurbain, pour nourrir leurs porcs. Le niveau de développement de la filière porcine dans chaque pays expliquerait cette différence. Au sud du Sénégal, les aliments industriels pour porcs sont commandés depuis Dakar (plus de 500 kilomètres de distance), ce qui renchérit leur coût et limite leur disponibilité et leur utilisation. Seuls les éleveurs ayant des ressources financières pouvaient s'approvisionner à Dakar où sont implantées les sociétés de provende. Ces unités de production d'aliment porcin sont encore peu nombreuses par rapport aux autres pays ouest-africains. En effet, en Côte d'Ivoire et au Bénin, des catégories d'aliment pour porc ont été développées pour répondre à leurs besoins selon le stade physiologique. L'élevage porcin en Afrique, notamment en Casamance, est généralement pratiqué en mode extensif et la conduite de l'alimentation des porcs réglée par le calendrier des activités agricoles (Agbokounou et al., 2016b).

\section{Conduite de la reproduction et productivité}

Lâge moyen à la mise à la reproduction et la taille moyenne par portée étaient respectivement de 7,6 mois et 7,4 porcelets. Ces résultats sont en accord avec ceux obtenus en Centrafrique (Abdallah-Nguertoum, 1997), au Tchad (Mopaté Logténé et al., 2010), au Sénégal (Missohou et al., 2001 ; Sambou, 2008), au Bénin (Ayssiwede et al., 2008), en Côte d'Ivoire (Tra Bi Tra, 2009) et dans d'autres pays africains (Agbokounou et al., 2016a). Cependant, la taille moyenne de la portée à la naissance était différente de celle rapportée par Umutoni (2012) au Burkina Faso (10 porcelets). La race des porcs étudiés peut être à l'origine de cette différence. En effet, notre étude a porté sur des élevages traditionnels où la race locale était prépondérante (90,4\% des exploitations) alors que dans celle d'Umutoni (2012), la principale race exploitée était la Large White (70\% des exploitations concernées).

Le sevrage survenait entre trois et six mois, le plus souvent à l'initiative de la truie $(86,1 \%)$. Ces résultats diffèrent de ceux d'Umutoni (2012) au Burkina Faso (66,5 jours), de la FAO (2012b) en République Démocratique du Congo (42 jours) et d'Ayssiwede et al. (2008) au Bénin (2,2 mois) en raison du système traditionnel majoritaire dans notre étude par rapport aux systèmes étudiés par ces auteurs. Les taux de mortalités avant et après sevrage des porcelets étaient proches de ceux trouvés par Agbokounou et al. (2016a) et nettement plus élevés que ceux notés par Buldgen et al. (1994). Pourtant, aucun cas de mortalité après sevrage n'a été déclaré au cours des enquêtes dans le système intensif. Ce résultat paraît peu plausible et s'apparenterait au manque d'informations dans l'état actuel des connaissances qui situe les mortalités postsevrage dans les élevages modernes entre 3 et $8 \%$ (Gaudré, 2011). La castration était pratiquée principalement entre trois et cinq mois dans $96,8 \%$ des élevages enquêtés. Ceci corrobore les résultats rapportés au Benin par Ayssiwede et al. (2008), et au Sénégal par Buldgen et al. (1994), qui sont respectivement de 3,6 mois et de quatre à cinq mois.

\section{Gestion sanitaire}

L'assistance médicale réservée au porc au sud du Sénégal est mitigée (faibles interventions des agents vétérinaires, quasi-absence de médicaments vétérinaires), ce qui favorise, d'une part, l'expansion de la médecine traditionnelle et, d'autre part, l'inaction des éleveurs en cas d'apparition de maladie. Ces observations rejoignent celles décrites par d'autres auteurs au Sénégal et en Gambie (Buldgen et al., 1994 ; Missohou et al., 2001 ; Sambou, 2008 ; Bassene, 2010 ; Secka, 2011) qui notent un faible niveau d'investissement dans le secteur de la santé. Ils sont, cependant, très différents de ceux d'Ayssiwede et al. (2008) au Bénin où $54,5 \%$ des éleveurs de porcs ont mis à jour des mesures prophylactiques pour préserver leurs élevages contre les maladies infectieuses. Cette différence serait due au contexte de réalisation des travaux de ces derniers. En effet, ils ont mené leur étude après l'épizootie de peste porcine africaine au Bénin en 1997, et cela dans le cadre d'un programme de relance de la filière porcine où les éleveurs ont été très sensibilisés et formés sur la gestion de l'élevage porcin.

\section{Commercialisation des porcs et rentabilité de l'élevage}

L'élevage traditionnel, système de production basé sur les pratiques rudimentaires (habitat, alimentation précaire, matériel, mode de reproduction), exploitait des porcs généralement de race locale générant un bénéfice net de $23 \%$ du prix de vente du porc mais avec des coûts de production moindres. L'élevage semi-intensif, système de production axé sur des pratiques d'élevage améliorées, employait des porcs de race mixte générant un bénéfice net de $14 \%$ du prix de vente du porc avec des charges assez importantes. Le système intensif, basé sur des pratiques modernes de production, exploitait surtout des porcs de race améliorée avec une taille moyenne du troupeau plus importante (28 porcs). Dans ce système, quatre fois plus de porcs étaient commercialisés par an que dans les systèmes précédents, pour un bénéfice net de $14 \%$ du prix de vente du porc avec des coûts de production très élevés. Ces écarts peuvent se justifier par les meilleures performances zootechniques (taille de la portée à la naissance, maitrise du sevrage, poids vif à la vente) et économiques. Les systèmes semi-intensif et intensif étaient surtout rencontrés dans la région de Ziguinchor où les salariés locaux et les particuliers étaient en grande majorité les principaux clients (75,4\%). Ces clients portaient une attention très particulière à la conformation des animaux et préféraient acheter les meilleurs sujets au poids vif.

Par ailleurs, on rencontre dans cette même région une association dynamique d'éleveurs de porcs qui les sensibilise sur l'importance et la nécessité de développer la filière porcine et d'harmoniser les méthodes et les prix de vente. Dans les deux autres régions, ces associations n'existent que de nom. Cette absence de collaboration entre producteurs justifierait la présence marquée de clients bissau-guinéens : 93,2 \% à Kolda et 83,9\% à Sédhiou, contre $20 \%$ à Ziguinchor.

En revanche, le bénéfice net obtenu (7566 FCFA) par porc élevé en système traditionnel sans considérer la main-d'œuvre familiale était légèrement supérieur à ceux des systèmes semi-intensif et intensif (respectivement 5435 et 6061 FCF/porc main-d'œuvre incluse). Cette particularité peut se justifier par les coûts élevés des aliments, des soins vétérinaires et la main-d'œuvre importante dans ces systèmes. Il va de soi que la considération de la main-d'œuvre réduirait ce bénéfice dans le système traditionnel. Dans ce sens, Le Goulven et al. (1999) ont remarqué que la marge par porc est plus importante lorsque certains facteurs de production proviennent de l'exploitation. Ainsi, la marge de 6304 FCFA/porc obtenue par ces auteurs dans un système de production où tout a été acheté correspond à celle du système intensif de notre étude.

Les études sur les performances économiques des exploitations porcines en Afrique de l'Ouest sont majoritairement focalisées sur les systèmes semi-intensif ou intensif (Ayssiwede et al., 2009 ; Tra Bi Tra, 2009 ; Umutoni, 2012). Dans ce cas, la marge brute annuelle varie entre 1 et 2 millions de francs CFA, ce qui est très supérieur aux nôtres (284 487 FCFA pour le système semi-intensif et 785109 FCFA pour le système intensif). Cette différence s'explique par le prix de vente, le poids à la vente et l'effectif des animaux vendus par an. Ces facteurs dépendent du niveau de développement du sous-secteur porcin dans chaque pays. Cette différence proviendrait aussi des approches méthodologiques, nos analyses économiques étant basées sur certaines valeurs théoriques issues d'extrapolation.

Pour le système traditionnel, rares sont les études qui ont pu déterminer sa rentabilité à cause de sa complexité, du manque de données et de sa conduite basée surtout sur la divagation. Certains auteurs préfèrent cibler les performances socioéconomiques de cet élevage (Mopaté Logténé et al., 2010) ou faire une étude longitudinale pour enregistrer les entrées et les sorties des exploitations pendant une certaine durée (Sambou, 2008 ; Doumana, 2001).

Les résultats de la présente étude sur le revenu net monétaire par porc charcutier dans les trois systèmes d'élevage ont été très inférieurs à ceux obtenus par Sambou (2008) dans la zone périurbaine de Dakar (20 080, 
22075 et 31943 FCFA respectivement pour les systèmes traditionnel, semi-intensif et intensif). Ces élevages ont recours tout au long de l'année au recyclage des déchets de la décharge de Mbeubeus alors que les coûts de l'alimentation dans la zone de notre étude étaient très élevés, surtout en période d'hivernage. Ils bénéficient également des prix de vente des porcs incitatifs en raison de leur proximité avec le plus grand centre de consommation du pays.

\section{CONCLUSION}

La présente étude, menée dans le cadre de la mise en œuvre du projet d'amélioration des productions porcines des élevages traditionnels de la Casamance naturelle, a montré que l'élevage de porc reste un soussecteur important dans la diversification des ressources alimentaires et monétaires. Cependant, les pratiques traditionnelles perdurent dans des proportions importantes.

L'élevage porcin, souvent associé à l'agriculture, est pratiqué par des femmes et des hommes majoritairement instruits. Les systèmes traditionnels d'élevage sont prédominants avec des pratiques de reproduction non contrôlée, une alimentation basée sur la divagation et dépendant de la disponibilité des déchets agroménagers. Au plan sanitaire, la faiblesse des services et du marché des médicaments vétérinaires se traduit par l'inaction ou l'emploi de traitements à base de produits traditionnels en cas de maladie. Les résultats économiques montrent que l'élevage porcin peut contribuer à lutter contre la pauvreté, et cette contribution serait d'autant plus importante si les contraintes qui le minent étaient levées. Dans les stratégies d'amélioration de ce secteur, il est capital d'assurer un encadrement technique des éleveurs à travers des formations sur la conduite et la gestion sanitaire du troupeau. En outre, il est important de trouver des voies de valorisation des ressources alimentaires localement disponibles pour atténuer les effets de la divagation et mieux organiser et renforcer la capacité des producteurs et des autres acteurs.

\section{Remerciements}

Les auteurs adressent leurs sincères remerciements au Fonds national des recherches agricoles et agro-alimentaires (FNRAA) du Sénégal pour avoir financé ce programme qui a été mis en œuvre par l'Institut sénégalais des recherches agricoles (ISRA) en collaboration avec l'Ecole inter-Etats des sciences et médecine vétérinaires (EISMV) de Dakar et le ministère de l'Elevage et des Productions animales (MEPA) du Sénégal.

\section{Contributions des auteurs}

WO, SBA et MD ont participé à la conception et à la planification de l'étude ; AM a participé à la conception de l'étude ; FN, RM et AED ont contribué à la collecte des données; WO, SBA, FN, RM et AED ont effectué les analyses statistiques et l'interprétation des données ; $\mathrm{FN}$ a rédigé la première version du manuscrit avec la contribution de WO et SBA ; tous les auteurs ont contribué à la révision du manuscrit ; $\mathrm{WO}, \mathrm{SBA}, \mathrm{MD}$ et $\mathrm{AM}$ ont révisé de manière critique le manuscrit.

\section{REFERENCES}

Abdallah-Nguertoum E., 1997. Elevage porcin en région périurbaine de Bangui (Centre Afrique). Thèse Doct. Vét., Ecole inter-Etats des sciences et médecine vétérinaires, Dakar, Sénégal, 111 p.

Agbokounou A.M., Ahounou G.S., Karim I.Y.A., Mensah G.A., Koutinhouin B., Hornick J.L., 2016a. Ethnologie et potentialités du porc local d'Afrique. J. Anim. Plant Sci., 29 (3) : 4665-4677

Agbokounou A.M., Ahounou G.S., Karim I.Y.A., Mensah G.A., Koutinhouin B., Hornick J.L., 2016b. Caractéristiques de l'élevage du porc local d'Afrique. J. Anim. Plant Sci., 30 (1) : 4701-4713

Ayssiwede S.B., Mankor A., Missohou A., Abiola F., 2009. Commercialisation et consommation de la viande de porc au Bénin. Rev. Afr. Santé Prod. Anim., 7 (2) : 105-112
Ayssiwede S.B., Missohou A., Abiola F., 2008. Les systèmes d'élevage de porcs au Bénin. Rev. Afr. Santé Prod. Anim., 6 (3-4) : 213-219

Bassene E.C., 2010. Etude typologique des élevages porcins de Jagoo (Dakar) et proposition d'une amélioration du cadre de vie des éleveurs. Thèse Doct. Vét., Ecole Inter-États des sciences et médecine vétérinaires, Dakar, Sénégal, 110 p.

Bathily P.B., 1975. Contribution à l'économie de la viande au Sénégal. Thèse Doct. Vét., Ecole inter-Etats des sciences et médecine vétérinaires, Dakar, Sénégal, 111 p.

Buldgen A., Piraux M., Dieng A., Schmit G., Compere R., 1994. Les élevages de porcs traditionnels du bassin arachidier Sénégalais. Rev. Mond. Zootech., $81: 63-70$

CIRAD, 2007. Populations porcines en Afrique de l'Ouest. http://pigtrop.cirad. $\mathrm{fr} / \mathrm{sp} /$ recursos/enciclopedia/les_porcs_du_monde/les_populations_porcines_ en_afrique_de_I_ouest (consulté 27 sept. 2015)

CSE, 2007. Caractérisation des systèmes de production agricole au Sénégal. Centre de suivi écologique, Dakar, Sénégal, 39 p., www.ntiposoft.com/ domaine_200/pdf/caractspasenegal.pdf (consulté 10 oct. 2016)

Doumana J.B., 2011. Effet de l'aménagement de bâtiment sur le cadre de vie, la productivité animale et la génération de revenus des éleveurs de porcs dans le quartier Jagoo (Dakar). Thèse Doct. Vét., Ecole inter-Etats des sciences et médecine vétérinaires, Dakar, Sénégal, 107 p.

Fall C.A., 2008. Deuxième rapport sur l'état des ressources phytogénétiques pour l'alimentation et l'agriculture dans le monde. ISRA, Dakar, Sénégal / FAO, Rome, Italie, www.fao.org/docrep/013/i1500e/Senegal.pdf (consulté 15 sept. 2017)

FAO, 2009. Le point sur l'élevage : changement dans le secteur de l'élevage (933). FAO, Rome, Italie, 25 p.

FAO, 2012a. Secteur porcin au Burkina Faso. Production et santé animales, FAO, Rome, Italie, 93 p. (Revues nationales de l'élevage ; 1)

FAO, 2012b. Secteur porcin en République démocratique du Congo. Production et santé animales, FAO, Rome, Italie, 85 p. (Revues nationales de l'élevage ; 2)

FAOSTAT, 2015. Base de données statistiques sur la production de l'élevage. http//faostat3.fao.org/browse/Q/QA/F (consulté 31 mai 2015)

Filmer D., Fox L., 2014. L'emploi des jeunes en Afrique subsaharienne. Washington, DC, Banque mondiale, 317 p. (Sér. Forum pour le développement de l'Afrique)

Gaudré D., 2011. Incidences des conditions d'élevage et d'alimentation en postsevrage sur les performances en engraissement. TechniPorc, 34 (1) : 21-26

Houndonougbo M.F., Adjolohoun S., Aboh B.A., Singbo A., Chrysotome C.A.A.M., 2012. Caractéristiques du système d'élevage porcin au Sud-Est du Bénin. Bull. Rech. Agron. Bénin (nº spécial Elevage \& Faune) : 15-21

Keiser A.M., 2004. Gestion financière, $7^{\mathrm{e}}$ Ed. ESKA, Paris, France, 622 p. (Coll. Gestion)

Lalèyê B.O., 2007. La filière porcine au Sénégal : commercialisation et consommation des viandes de porc et de phacochère dans les départements de Dakar, Fatick, Ziguinchor et Kolda. Thèse Doct. Vét., Ecole inter-Etats des sciences et médecine vétérinaires, Dakar, Sénégal, 130 p.

Le Glaumec L.G.A.L., 2006. Etude épidémiologique du cycle sauvage de la peste porcine africaine dans la région du Sine Saloum au Sénégal. Thèse Doct. Vét., Ecole vétérinaire, Toulouse, France, 122 p.

Le Goulven K., Boutonnet J.P., Codron J.M., 1999. Marketing an agricultural production in a "transition" economy: pork marketing chain from Nam Thanh to Hai Phong (Vietnam). Rev. Elev. Med. Vet. Pays trop., 52 (3-4): $305-$ 312, doi : 10.19182/remvt.9679

Mankor A., 2009. Consommation urbaine de viande en Afrique de l'Ouest : I'exemple de Dakar. Grain Sel (46-47) : 16-17

Mbetid-Bessane E., Gafsi M., 2003. Faiblesse de la main-d'œuvre familiale et diversification des activités dans les exploitations agricoles de la zone cotonnière en Centrafrique : quel enseignement pour le conseil de gestion aux agriculteurs ? In : Actes Colloq. Savanes africaines : des espaces en mutation, des acteurs face à de nouveaux défis, Garoua, Cameroun, mai 2002 (éds Jamin J.-Y., Seiny Boukar L., Floret C.). Prasac, N’Djamena, Tchad / Cirad, Montpellier, France, 7 p.

MEPA, 2015. Statistiques des productions et importations des produits animaux au Sénégal en 2014. Ministère de l'Elevage et des Productions animales, Direl, Cellules des études et de la planification, Dakar, Sénégal, 7 p.

Missohou A., Niang M., Foucher H., Dieye P.N., 2001. Les systèmes d'élevage porcin en Basse Casamance (Sénégal). Note de recherche. Cah. Agric., 10 : 405-408 
Mopaté Logténé Y., Koussou M.O., 2003. L'élevage porcin, un élevage ignoré mais pourtant bien implanté dans les agro-systèmes ruraux et périurbains du Tchad. In : Actes Colloq. Savanes africaines : des espaces en mutation des acteurs face à de nouveaux défis, Garoua, Cameroun, mai 2002 (éds Jamin J.-Y., Seiny Boukar L., Floret C.). Prasac, N'Djamena, Tchad / Cirad, Montpellier, France, 9 p.

Mopaté Logténé Y., Koussou M.O., Nguertoum E.A., Ngo Tama A.C., Lakouténé T., Awa D.N., Mal Mal H.E., 2010. Caractéristiques et performances des élevages porcins urbains et périurbains des savanes d'Afrique centrale : cas des villes de Garoua, Pala et Bangui. Actes Colloq. Savanes africaines en développement : innover pour durer, Garoua, Cameroun, 20-23 avr. 2009 (éds Seinyboukar L., Boumard P.). Cirad, Montpellier, France

Ndebi G., Kamajou J., Ongla J., 2009. Analyse des contraintes au développement de la production porcine au Cameroun. Tropicultura, 27 (2) : 70-76

Niang M., Mbaye M., 2013. Evolution des exportations de bétail malien au Sénégal suite aux récentes crises. Rapport final. APCAM/MSU/USAID, 43 p. http://fsg.afre.msu.edu/promisam_II.2/Etude_exportation_b \%C3 \%A9tail Mali_S \%C3 \%A9n \%C3 \%A9gal_Rapport_final.pdf (consulté 13 janv. 2017)

Ossebi W., Ayssiwede S.B., Nimbona F., Malou R., Djettin A.E., Diop M., Missohou A., 2018. Obstacles to the development of the pig value chain in Casamance (Senegal): what do actors of the subsector say? Rev. Elev. Med. Vet. Pays trop., 71 (1-2): 15-22, doi: 10.19182/remvt.31286

Porphyre V., 2009. Enjeux et contraintes des filières porcines en Afrique de I'Ouest. Grain Sel (46-47) : 26-27

\section{Summary}

Ossebi W., Ayssiwede S.B., Nimbona F., Malou R., Djettin A.E., Diop M., Missohou A. Zootechnical and economic analysis of pig farming systems in Casamance (Senegal)

To understand better the zootechnical and economic characteristics of pig farming, a diagnostic study was conducted on 324 pig farms from March to June 2016, in the form of cross-sectional and retrospective surveys in the three regions of Southern Casamance, Senegal. The results showed that pig farming was practiced by both married and educated men and women. They were mainly of the Diola, Mancagne and Balante ethnic groups and combined pig breeding with agriculture, private activity or trade. They operated in three different farming systems (traditional, semi-intensive or intensive), with a marked predominance of the traditional system (85.5\% of farms). The latter was characterized by improved traditional hog barns $(51.1 \%)$ or makeshift sheds $(28.4 \%)$, where mostly local swine $(90.4 \%$ of farms) were bred. The average size of the herd was $19.2 \pm 20.1$ pigs. In $97 \%$ of cases, breeders fed the animals from kitchen waste, and agricultural and agro-industrial by-products. The mean age at breeding was $7.6 \pm 1.8$ months and the mean litter size was $7.4 \pm 2.2$ piglets. This livestock subsector has a real poverty alleviation potential because it generates net incomes of 5435 to 7566 FCFA per fattened pig per year, depending on the system. Nevertheless, the lack of training of livestock farmers, inadequate pig housing, and feed and health constraints remain the main factors hindering the development of pig farming in the region. It would be useful to find alternative ways to improve housing conditions, feeding and health monitoring of pigs, along with better organization and capacity building of producers and other actors in the pig sector.

Keywords: swine, farming system, commercialization, zootechny, Senegal
Sambou G., 2008. Analyse des impacts de la décharge de Mbeubeuss (Dakar) sur les élevages porcins environnants. Thèse Doct. Vét., Ecole inter-Etats des sciences et médecine vétérinaires, Dakar, Sénégal, 138 p.

Seck I., 2007. Epidémiologie de la peste porcine africaine au Sénégal estimation de la prévalence de la maladie dans les régions de Fatick, Kolda et Ziguinchor. Thèse Doct. Vét., Ecole inter-Etats des sciences et médecine vétérinaires, Dakar, Sénégal, 119 p

Secka A., 2011. Pig production system, marketing chain and cysticercosis awareness in the Gambia and Senegal. Bull. Anim. Health. Prod. Afr., 59: 459-469

Smith P., 2013. Sampling and estimation for business surveys. In: Designing and conducting business surveys (Eds. Snijkers G., Haraldsen G., Jones J., Willimack D.). Wiley, NJ, USA, 165-218, doi: 10.1002/9781118447895.ch05

Tra Bi Tra C., 2009. Filière porcine en Côte d'Ivoire : production, propositions d'amélioration et perspectives de développement. Thèse Doct. Vét., Ecole inter-Etats des sciences et médecine vétérinaires, Dakar, Sénégal, 146 p.

Umutoni C., 2012. Evaluation technico-économique des élevages de porc à Bobo-Dioulasso (Burkina Faso). Mémoire Master Prod. anim. dév. durable, Ecole inter-Etats des sciences et médecine vétérinaires, Dakar, Sénégal, 45 p.

Youssao A.K.I., Koutinhouin G.B., Kpodekon T.M., Bonou A.G., Adjakpa A., Dotcho C.D.G., Atodjinou F.T.R., 2008. Pig production and indigenous genetic resources in suburban areas of Cotonou and Abomey-Calavi in Benin. Rev. Elev. Med. Vet. Pays trop., 61 (3-4): 235-243, doi: 10.19182/ remvt.9995

\section{Resumen}

Ossebi W., Ayssiwede S.B., Nimbona F., Malou R., Djettin A.E., Diop M., Missohou A. Análisis zootécnico y económico de los sistemas de producción porcina en Casamance (Senegal)

Para entender mejor las características zootécnicas y económicas de la producción porcina, se llevó a cabo un estudio diagnóstico en 324 fincas porcinas, de marzo a junio 2016, con un formato de encuestas transversales y retrospectivas en tres regiones del sur de Casamance, Senegal. Los resultados mostraron que la producción porcina fue practicada por hombres y mujeres casados y educados. Pertenecieron principalmente a los grupos étnicos Diola, Mancagne y Balante y combinaron la cría de cerdos con agricultura, y actividad privada o comercio. Operaron en tres sistemas finqueros diferentes (tradicional, semi-intensivo o intensivo), con predominancia marcada del sistema tradicional (85,5\% de las fincas). Este se caracterizó por establos porcinos tradicionales mejorados $(51,1 \%)$ o cobertizos improvisados (28,4\%), donde se criaron principalmente cerdos locales $(90,4 \%$ de las fincas). El tamaño promedio del hato fue 19,2 $\pm 20,1$ cerdos. En 97\% de los casos, los criadores alimentaron a los animales con deshechos de cocina y subproductos agrícolas y agroindustriales. La edad promedio a la cría fue de $7,6 \pm 1,8$ meses y el tamaño promedio de la camada fue de 7,4 $\pm 2,2$ lechones. Este sub sector ganadero presenta un verdadero potencial de alivio de la pobreza, ya que genera ingresos netos de 5435 a 7566 FCFA por cerdo de engorde por año, según el sistema. Sin embargo, la falta de capacitación de los productores ganaderos, el albergue inadecuado de los cerdos y obstáculos alimenticios y de salud representan aun los principales factores que impiden el desarrollo de la ganadería porcina en la región. Seria útil encontrar formas alternativas para mejorar las condiciones de alojamiento, de alimenticio y de seguimiento de salud de los cerdos, junto con una mejor organización y desarrollo de capacidades de los productores y otros actores en el sector porcino.

Palabras clave: cerdo, sistemas de explotación, comercialización, zootecnia, Senegal 